\title{
Relaciones filogenéticas de Rhodolirium montanum Phil. y especies afines, basadas en secuencias nucleotídicas de la región ITS y análisis cariotípico
}

\section{Phylogenetic relationships of Rhodolirium montanum Phil. and related species based on nucleotide sequences from ITS region and karyotype analysis}

\author{
Manuel Muñoz ${ }^{1,2}$, Ricardo Riegel ${ }^{1}$, Peter Seemann $^{1}$, Patricio Peñallillo $^{3}$, Flavia Schiappacasse ${ }^{4} \&$ \\ José NúÑEZ ${ }^{5}$ \\ ${ }^{1}$ Instituto de Producción y Sanidad Vegetal, Universidad Austral de Chile, Casilla 567, Valdivia, Chile. \\ Escuela de Graduados, Facultad de Ciencias Agrarias, Universidad Austral de Chile, Casilla 567, Valdivia, Chile. \\ ${ }^{3}$ Instituto de Biología Vegetal y Biotecnología, Universidad de Talca, 2 Norte 685, Talca, Chile. \\ ${ }^{4}$ Departamento de Horticultura, Facultad de Ciencias Agrarias, Universidad de Talca, 2 Norte 685, Talca, Chile. \\ ${ }^{5}$ Instituto de Zoología, Facultad de Ciencias, Universidad Austral de Chile, Casilla 567, Valdivia, Chile. \\ manuelmun@gmail.com
}

\section{RESUMEN}

La taxonomía de los géneros chilenos de Amaryllidaceae es confusa, persistiendo problemas taxonómicos tanto a nivel de géneros como de especies. Actualmente existe controversia acerca de la correcta clasificación de especies hasta ahora asignadas a Rhodophiala C.Presl. A través del estudio de aspectos morfológicos, recientemente se ha sugerido la rehabilitación del género Rhodolirium Phil., donde Rhodolirium montanum Phil. es considerada como especie tipo. Esta especie ha sido denominada Rhodophiala rhodolirion (Baker) Traub en las clasificaciones tradicionales. Dado que los datos morfológicos han provisto información limitada y ambigua en la clasificación de las Amarilidáceas chilenas, este trabajo evalúa la pertinencia de formalizar la rehabilitación del género Rhodolirium y en particular de la especie Rhodolirium montanum, a través de un análisis de morfología cromosómica y de secuencias nucleotídicas de la región ITS de seis especies nativas de Amaryllidaceae. Los datos moleculares indican que las especies Rhodophiala bagnoldii, $R$. montana, $R$. splendens, $R$. phycelloides y $R$. ananuca forman un grupo monofilético más emparentado con Hippeastrum Herb. que con Rhodolirium, género que se presenta como grupo hermano de Phycella en el análisis filogenético. La separación de Rhodolirium con respecto de Rhodophiala está respaldada por diferencias en número y morfología cromosómica, presentando el primero un número cromosómico de $2 \mathrm{n}=16$ y un índice de asimetría intracromosómica (Isc) de 0,46; mientras que el segundo posee $2 \mathrm{n}=18$ e Isc de $0,59-0,62$. De esta manera, los resultados de los estudios cromosómicos y moleculares, junto a diferencias en exomorfología, tales como forma del estigma, perigonio y paraperigonio, apoyan la validez del género Rhodolirium Phil. y por ende de la especie Rhodolirium montanum Phil.

Palabras Clave: Rhodophiala, Rhodolirium montanum, Amaryllidaceae, ITS, cariotipo.

\begin{abstract}
The taxonomy of Chilean Amaryllidaceae is confusing. Taxonomic problems still persist at the level of genera and species. Currently there is controversy about the correct classification of species so far assigned to Rhodophiala C.Presl. Through the study of morphological characters, recently has been suggested the rehabilitation of the genus Rhodolirium Phil., where the species Rhodolirium montanum Phil. is considered as type species. This species was named Rhodophiala rhodolirion (Baker) Traub in traditional classifications. Since morphological data have provided limited and ambiguous information in the classification of Amaryllidaceae in Chile, this paper assesses the relevance of formalize the rehabilitation of genus Rhodolirium and particularly the resurrection of the species R. montanum, through an analysis of chromosomal morphology and nucleotide sequences of the ITS region of six native species of Amaryllidaceae. The molecular data indicate that the species Rhodophiala bagnoldii, $R$. montana, $R$. splendens, $R$. phycelloides and $R$. ananuca form a monophyletic group more closely related to Hippeastrum than Rhodolirium. This genus is presented as closely related to Phycella in the phylogenetic analysis using ITS sequences. This separation of Rhodolirium with respect Rhodophiala is supported by differences in chromosome number and morphology, presenting the first, chromosome number of $2 \mathrm{n}=$ 16 and intrachromosomal asymmetry index (Isc) of 0.46 , while the latter has $2 \mathrm{n}=18$ and Isc of 0.59 to 0.62 . Thus,
\end{abstract}


chromosomal and molecular results together differences in exomorphology already mentioned by other authors support the rehabilitation of the genus Rhodolirium Phil. and the resurrection of the species Rhodolirium montanum Phil.

KEYworDs: Rhodophiala, Rhodolirium montanum, Amaryllidaceae, ITS, karyotype.

\section{INTRODUCCIÓN}

La familia Amaryllidaceae comprende aproximadamente 60 géneros y alrededor de 850 especies distribuidas principalmente en las zonas cálidas, cuyos centros de diversificación se encuentran en Sudamérica, Sudáfrica y la región mediterránea de Europa (Meerow \& Snijman 1998, Meerow et al. 2000a, Strydom 2005). Esta familia de geófitas bulbosas se destaca por sus numerosos géneros cultivados como ornamentales, entre los que se encuentran Amaryllis L., Crinum L., Galanthus L., Leucojum L., Lycoris Herb. y Narcissus L. (Simpson 2006). Los géneros Placea Miers y Phycella Lindl., endémicos de Chile y el género nativo Rhodophiala C.Presl, presentan vistosas flores, por lo que han llamado la atención de los investigadores en floricultura dado su potencial ornamental (Schiappacasse et al. 2002, Olate \& Bridgen 2005). No obstante lo anterior, entre los problemas taxonómicos más controvertidos de la familia está la falta de consenso en las clasificaciones tribales y en las delimitaciones genéricas (Meerow et al. 1999, 2000a, 2000b) debido principalmente a la compleja variación morfológica de géneros tales como Hippeastrum Herb., Phycella y Rhodophiala (Arroyo \& Cutler 1984).

En Chile, la taxonomía de Amaryllidaceae no ha estado exenta de confusiones y aun persisten problemas taxonómicos tanto a nivel de géneros como de especies (Ravenna 2003). Ejemplo de lo anterior es que, por muchos años, las especies de Amaryllidaceae de corola vistosa e infundibuliforme y con estigma capitado o trífidos fueron consideradas tanto dentro del género Hippeastrum como de Rhodophiala (Marticorena \& Quezada 1985). Basándose principalmente en caracteres morfológicos, Ravenna (2003) propone una nueva clasificación para los géneros chilenos de la familia Amaryllidaceae, citando 11 géneros y alrededor de 35 especies. Sin embargo, la simple delimitación morfológica de los géneros de la familia es difícil de establecer por la compleja variabilidad de los caracteres utilizados hasta ahora (Baeza et al. 2009).

Baeza \& Schrader (2004) y Baeza et al. (2006, 2007a, 2007b, 2009) indican la importancia de los estudios cariológicos para clarificar la posición taxonómica de los géneros de Amaryllidaceae chilenos. Anteriormente, otros investigadores ya habían señalado la significancia del número y forma de los cromosomas en la clasificación genérica de esta familia (Naranjo \& Andrada 1975, Naranjo \& Poggio 2000, Meerow et al. 2000a, Palma-Rojas 2000).

El género Rhodolirium descrito por Philippi (1858), por muchos años estuvo inmerso en géneros, tales como Hippeastrum y Rhodophiala. Naranjo \& Poggio (2000) propusieron la rehabilitación del género Rhodolirium sobre la base del número y morfología de los cromosomas. Ravenna (2003) acepta este género utilizando el nombre Rhodolirion, no existiendo una explicación clara para este cambio de nombre. Sin embargo, éste es un nombre utilizado por Baker (1878) cuando denominó las especies descritas por Philippi como sinónimos de Hippeastrum. También, Baker renombró a R. andinum como Hippeastrum rhodolirion. Posteriormente, en 1888, el mismo autor empleó Rhodolirion como un subgénero de Hippeastrum, considerando erradamente $R$. andinum como el tipo.

Desde el punto de vista morfológico, Rhodolirium incluye plantas uni- a plurifloras, de perigonio infundibuliforme y tubo alargado, el paraperigonio, si está presente, es formado por lacinias o escamas libres, y el estigma es capitado. Este género comprende a cinco especies, las cuales se distribuyen desde el norte de Chile, en zonas de vegetación de neblina costera, hasta la provincia de Osorno por el sur, y desde el cinturón costero hasta el lado oriental de los Andes, en Argentina, a alturas entre 150-2500 m s.n.m. (Ravenna 2003).

Bajo los nuevos paradigmas de la taxonomía integrativa (Dayrat 2005), los datos morfológicos deben ser sometidos al filtro de otras aproximaciones y tipos de datos diferentes, entre ellos, la información a nivel molecular. Una de las regiones del genoma cuya variación es una valiosa fuente de caracteres informativos para la inferencia filogenética en Angiospermas, es la región del espaciador interno transcrito (ITS) del ADN ribosomal nuclear 18S-26S (Baldwin et al. 1995). Meerow et al. (2000b), utilizando datos provenientes del análisis de la región ITS del cistrón ribosomal nuclear 18S-5,8S-26S, distinguen a Rhodophiala de Hippeastrum y sostienen que Rhodophiala tendría un origen aparentemente polifilético. Además, el género Phycella aparecía como el grupo hermano a éstos y otros géneros americanos.

Puesto que el estudio de la región ITS ha resultado 
resolutivo en la delimitación de grupos filogenéticamente más consistentes dentro de los géneros americanos de la familia Amaryllidaceae, los objetivos del presente estudio son determinar las relaciones de parentesco de cinco especies chilenas del género Rhodophiala y una única especie del género Rhodolirium, con respecto a otras especies de géneros afines cuyas secuencias ITS ya han sido publicadas e integrar a esta hipótesis de relaciones la información citológica de las especies y las evidencias provenientes de la exomorfología.

\section{MATERIAL Y MÉTODOS}

Material Vegetal

Rhodophiala bagnoldii (Herb.) Traub y R. phycelloides (Herb.) Hunz. fueron recolectadas en Huasco, costa de la Región de Atacama; R. ananuca (Phil.) Traub en Aguada de Tongoy, costa de la Región de Atacama; R. montana (Phil.) Traub en Laguna del Maule, en la cordillera de la Región del Maule, R. splendens (Rengifo) Traub en Vilches Alto, cordillera de la Región del Maule y Rhodolirium montanum en Los Queñes, cordillera de la Región del Maule. Los ejemplares herborizados de cada una de estas especies fueron depositados en el herbario de la Universidad de Talca con los números 3357, 2407, 3358, 2269, 3015 y 2679, respectivamente. Además, se mantienen en cultivo bulbos de las especies mencionadas bajo invernadero frío en la Estación Experimental de Panguilemo de la misma institución. El material fue identificado utilizando las claves de identificación propuestas por Traub \& Moldenke (1949) y Traub (1956) y posteriormente comparado con las descripciones originales.

Los caracteres morfológicos utilizados para confeccionar la Tabla II se observaron en ejemplares frescos y especímenes herborizados, además de consultar la literatura respectiva, Muñoz y Moreira (2000) y Ravenna (2003).

\section{AnÁlisis CROMosómicos}

Para las especies mencionadas de Rhodophiala, a excepción de $R$. phycelloides, se realizaron preparaciones citológicas a partir de ápices radicales según el protocolo descrito por Grant et al. (1984) y adaptado por Muñoz et al. (2006).

Se tomaron cinco placas metafásicas provenientes de 2 a 4 plantas para cada especie. Se contabilizó el número cromosómico somático y para cada cromosoma se midió la longitud del brazo largo (BL) y del brazo corto (BC). Los cromosomas se agruparon en pares de acuerdo a su similitud y se ordenaron de mayor a menor tamaño. Con estas mediciones se calculó el índice centromérico (BC/LT x 100), fórmula cariotípica básica (Levan et al. 1964) y el índice de asimetría cariotípica (A1, intracromosómica) según la fórmula propuesta por Romero Zarco (1986).
ANÁLISIS MOLECULARES

La extracción de ADN se realizó a partir de $0.1 \mathrm{~g}$ de hojas jóvenes que fueron maceradas en $500 \mu \mathrm{L}$ de buffer de extracción CTAB 2\% (Tris $100 \mathrm{mM} \mathrm{pH} \mathrm{8,} \mathrm{EDTA} 20 \mathrm{mM}$, $\mathrm{NaCl} 1,4 \mathrm{M}, \mathrm{CTAB} 2 \%$ ) y $2 \mu \mathrm{L}$ de mercaptoetanol. Se incubó durante 30 minutos a $60^{\circ} \mathrm{C}$ y luego se adicionó $500 \mu \mathrm{L}$ de cloroformo:fenol:alcohol isoamílico (25:24:1) seguido de centrifugado ( 5 min a $10.000 \mathrm{rpm}$ ). Después de la adición de $200 \mu \mathrm{L}$ de isopropanol frío $\left(-18^{\circ} \mathrm{C}\right)$ al sobrenadante, se centrifugó nuevamente (10 $\mathrm{min}$ a $11.000 \mathrm{rpm})$, se lavó el precipitado con etanol frío $70 \%$ y se resuspendió en agua deionizada estéril.

La región ITS fue amplificada utilizando los partidores ITS1-Plant-F, (5-CGCGAGAAGTCCACTG-3)' e ITS C26A-R (5'GTTTCTTTTCCTCCGCT-3') (Varghese et al. 2003). La amplificación se llevó a cabo en un volumen total de $50 \mu \mathrm{L}$, consistente de $35,2 \mu \mathrm{L}$ de agua deionizada estéril, $5 \mu \mathrm{L}$ de buffer 10X (Fermentas), $1,25 \mu \mathrm{L}$ de dNTPs ( $2 \mathrm{mM}$; Invitrogen), $3 \mu \mathrm{L}$ de $\mathrm{MgCl}_{2}$ ( $25 \mathrm{Mm}$; Fermentas), $1 \mu \mathrm{L}$ de cada partidor $(10 \mathrm{pmol} / \mu \mathrm{L})$ y $0,1 \mu \mathrm{L}$ de Taq polimerasa $(5 \mathrm{U} / \mu \mathrm{L})$ (Fermentas). Las amplificaciones se realizaron utilizando el siguiente perfil térmico: denaturación inicial a $94{ }^{\circ} \mathrm{C}$ por 3 min., para luego realizar 39 ciclos de $94{ }^{\circ} \mathrm{C}$ por $30 \mathrm{seg}$., reasociación a $50^{\circ} \mathrm{C}$ por $1 \mathrm{~min}$. y extensión a $72{ }^{\circ} \mathrm{C}$ por $1 \mathrm{~min}$. Se finalizó con una extensión final a 72 ${ }^{\circ} \mathrm{C}$ por $5 \mathrm{~min}$. Los productos fueron visualizados mediante electroforesis en geles de agarosa al 1,5\% mediante tinción con bromuro de etidio $(0,5 \mu \mathrm{g} / \mathrm{mL})$.

Todos los productos amplificados fueron purificados y secuenciados por ambos extremos (Macrogen Inc., Seul, Corea). El alineamiento de las secuencias se hizo con el programa MAFFT 5.0 (Katoh et al. 2005) bajo métodos de refinamiento iterativos, incorporando la información del alineamiento más seguro tanto local (L-INS-i y EINSi) como global (G-INS-i). Se usaron los parámetros por defecto involucrados en cada algoritmo. Las secuencias fueron depositadas en Genbank con los siguientes números de accesión para cada especie: Rhodophiala montana (Genbank, FN436271), R. splendens (FN436273), $R$. bagnoldii (FN436270), $R$. phycelloides (FN436272), $R$. ananuca (FN386450) y Rhodolirium montanum (FN436274).

\section{ANÁLISIS FILOGENÉTICO}

LOS ANÁUSIS DE M ÁXIM A VEROSIM IUTUD (ML) SE REALZARON CON GARLI 0.951 (ZWICKL 2006). LA ELECCIÓN DEL M ODELO DE mejor ajuste fue basado en el CRIterio de Akaike, AIC (Posada \& Buckiey 2004), usando el P Rogram a JModelTest (Posada 2008). Los análsis fueron finalzados después DE 10000 SIN UN MEJORAM IENTO DE LA TOPOLOGÍA DEL ÁRBOL Para asegurar ConVergencia SE reALZARon dos análsis dE ML. LOS SOPORIES DE CADA NODO FUERON EVALUADOS USANDO 200 ITERACIONES POR BOOTSTRAP (FELSENSTEIN 1985), CON CADA ITERACIÓN TERM INADA DESPUÉS DE 5000 GENERACIONES SIN 
un mejoramiento significativo de la topología. Se realizó también un análisis Bayesiano usando MrBayes 3.0b4 (Ronquist \& Huelsenbeck 2003). Al igual que en el análisis de ML, la elección del modelo basado en AIC se hizo con jModelTest (Posada 2008). Las cadenas de Markov Monte Carlo (MCMC) fueron corridas por 7 x $10^{6}$ generaciones. Los árboles se muestrearon cada cien generaciones. La longitud de las ramas de los árboles fueron salvadas y el 25\% del "burn-in" descartado después de graficar el logaritmo de los valores de verosimilitud de los árboles muestreados contra el número de generaciones.

En la construcción de los filogramas se utilizó como grupo externo Amaryllis belladona L. y A. paradisicola Snijman, cuyas secuencias ITS se encuentran en la base de datos Genbank con números de acceso AF373084 y AF373073, respectivamente. Además, se agregaron las secuencias de Rhodophiala bifida (Herb.) Traub (AF223477) R. chilensis (L'Hér.) Traub (AF223480), Phycella ignea (Lindl.) Lindl. (AF223505) y de Hippeastrum brasilianum (Traub \& J.L.Doran) Dutilh (AF223479), H. macbridei (Vargas) Gerea \& Brako (AF223509), H. mollevilquense (Cárdenas) Van Scheepen (AF223477), e H. parodii Hunz. \& A.A.Cocucci (AF223508).

\section{RESULTADOS}

ANÁLISIS CROMOSÓMICOS, FÓRMULA CARIOTÍPICA E ÍNDICES DE ASIMETRÍA

De las seis especies en estudio, cinco mostraron número cromosómico somático de $2 \mathrm{n}=18$; sólo Rhodolirium montanum difiere de las demás y presenta $2 \mathrm{n}=16$ (Fig. 1). Los índices de asimetría de los cariotipos tienden a acercarse a 0,6 en la mayoría de las especies, diferenciándose aquél de $R$. montanum, que es de 0,46. En cuatro especies, los dos pares de cromosomas más pequeños son metacéntricos, los de mayor tamaño son submetacéntricos y subtelocéntricos (Fig. $1 \mathrm{~A}, \mathrm{~B}, \mathrm{C}$ ), en $R$. montanum los 2 pares más pequeños son subtelocéntricos y metacéntricos, los de mayor tamaño son metacéntricos, submetacéntricos y subtelocéntricos (Fig. 1D y Tabla I).

ANÁlisis DE SECUENCIAS DE LA REGIÓN ITS

El filograma de la Figura 2 representa las relaciones filogenéticas entre las especies analizadas en este estudio. Es posible observar dos clados con buen soporte de bootstrap $(\mathrm{BS}=100)$ y de probabilidad a posteriori $(\mathrm{PP}=1.0)$. En el clado inferior se forman dos subclados

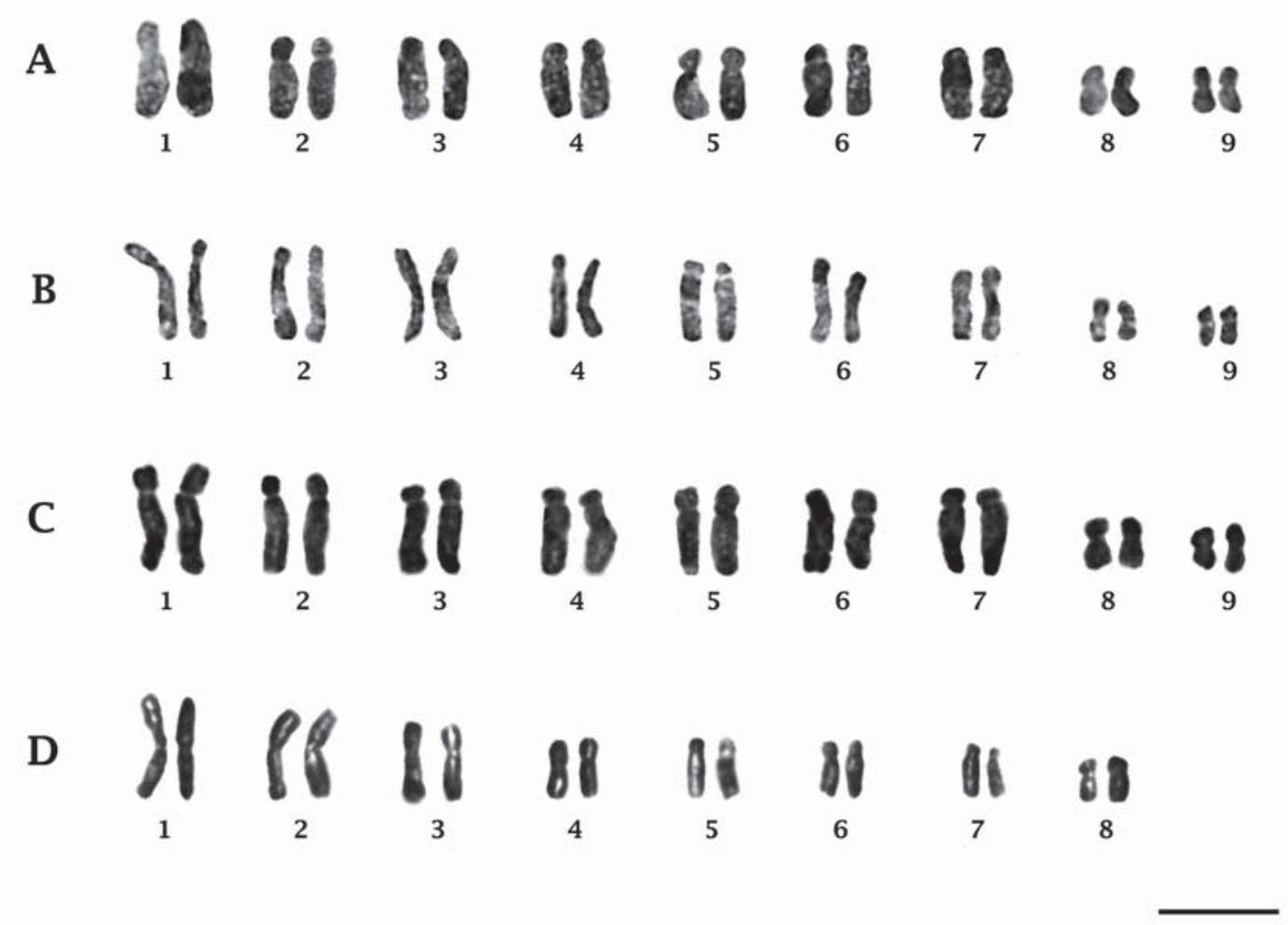

Figura 1. Cariotipos: A. Rhodophiala bagnoldii, B. Rhodophiala montana, C. Rhodophiala splendens, D. Rhodolirium montanum. Barra $=10 \mu \mathrm{m}$.

Figure 1. Karyotypes: A. Rhodophiala bagnoldii, B. Rhodophiala montana, C. Rhodophiala splendens, D. Rhodolirium montanum. Bar= $10 \mu \mathrm{m}$. 
con buenos soportes $(\mathrm{BS}=100$ y $\mathrm{PP}=1.0)$. Un subclado incluye a todas las especies del género Hippeastrum cuyo número cromosómico diploide es 22 y el otro subclado a las especies pertenecientes al género Rhodophiala con un $2 \mathrm{n}=18$.

En el clado superior se ubican dos subclados con buen apoyo ( $\mathrm{BS}=100$ y $\mathrm{PP}=1.0)$, separándose el género Phycella de Rhodolirium. Rhodolirium montanum difiere en $15,5 \%$ de sus bases con respecto a las especies de Rhodophiala (640 sitios alineados). Esto se ve respaldado por diferencias tanto en su cariología $(2 n=16)$ como en su morfología (Tabla II) con respecto al género Rhodophiala.

TABLA I. Caracterización morfológica de los cromosomas de distintas especies de Rhodophiala y Rhodolirium montanum (=Rhodophiala rhodolirion).

TABLE I. Characterization of chromosomal morphology of different species of Rhodophiala and Rhodolirium montanum (=Rhodophiala rhodolirion).

\begin{tabular}{|c|c|c|c|c|}
\hline ESPECIE & $2 n$ & PLOIDÍA & FóRMULA CARIOTÍPICA* & $\begin{array}{l}\text { ÍNDICE DE ASIMETRÍA } \\
\text { INTRACROMOSÓMICA }\end{array}$ \\
\hline R. montana & 18 & $2 X$ & $\begin{array}{c}<10 \%: 4 \mathrm{~m} \\
>10 \%: 6 \mathrm{sm}+8 \mathrm{st}\end{array}$ & 0,59 \\
\hline R. splendens & 18 & $2 \mathrm{X}$ & $\begin{array}{c}<10 \%: 4 \mathrm{~m} \\
>10 \%: 8 \mathrm{sm}+6 \mathrm{st}\end{array}$ & 0,62 \\
\hline R. bagnoldii & 18 & $2 \mathrm{X}$ & $\begin{array}{c}<10 \%: 2 \mathrm{~m} \\
>10 \%: 8 \mathrm{sm}+6 \mathrm{st}\end{array}$ & 0,60 \\
\hline R. ananиса & 18 & $2 X$ & $\begin{array}{c}<10 \%: 4 \mathrm{~m} \\
>10 \%: 6 \mathrm{sm}+8 \mathrm{st}\end{array}$ & 0,61 \\
\hline R. montanum & 16 & $2 \mathrm{X}$ & $\begin{array}{c}<10 \%: 2 \mathrm{st}+2 \mathrm{sm} \\
>10 \%: 6 \mathrm{~m}+4 \mathrm{sm}+2 \mathrm{st}\end{array}$ & 0,46 \\
\hline
\end{tabular}

*El complemento haploide se dividió en dos grupos de cromosomas: cromosomas pequeños, que individualmente representan menos del $10 \%$ del complemento haploide y cromosomas grandes, que individualmente representan más del 10\%. Cromosomas metacéntricos (m), submetacéntricos ( $\mathrm{sm}$ ) y subtelocéntricos (st). / The haploid complement was divided into two groups of chromosomes: chromosomes small, that individually represent less than $10 \%$ of the haploid chromosome complement and large, that individually represent more than $10 \%$. Metacentric (m), submetacentric ( $\mathrm{sm})$ and subtelocentric (st).

TABLA II. Caracteres morfológicos de los géneros Rhodolirium, Phycella, Rhodophiala, Placea y Traubia.

TABLE II. Morphological characters for Rhodolirium, Phycella, Rhodophiala, Placea and Traubia.

\begin{tabular}{|c|c|c|c|}
\hline GÉnERos & Forma del Perigonio & Forma DEL Estigma & PARAPERIGONIO \\
\hline Rhodolirium & Infundibuliforme & Capitado-trilobado & $\begin{array}{l}\text { Si presente formado por } \\
\text { apéndices escamosos libres }\end{array}$ \\
\hline Phycella & Tubuloso & Capitado & $\begin{array}{l}\text { Si presente formado por } \\
\text { apéndices fimbriados libres }\end{array}$ \\
\hline Rhodophiala & Infundibuliforme & Trífido & $\begin{array}{l}\text { Si presente formado por } \\
\text { apéndices fimbriados libres }\end{array}$ \\
\hline Placea & Infundibuliforme & Trilobado & $\begin{array}{c}\text { Presente formado por } \\
\text { apéndices unidos en la } \\
\text { base o en toda su longitud } \\
\text { (corona) }\end{array}$ \\
\hline Traubia & Estrellado & Capitado punteado & Ausente \\
\hline
\end{tabular}




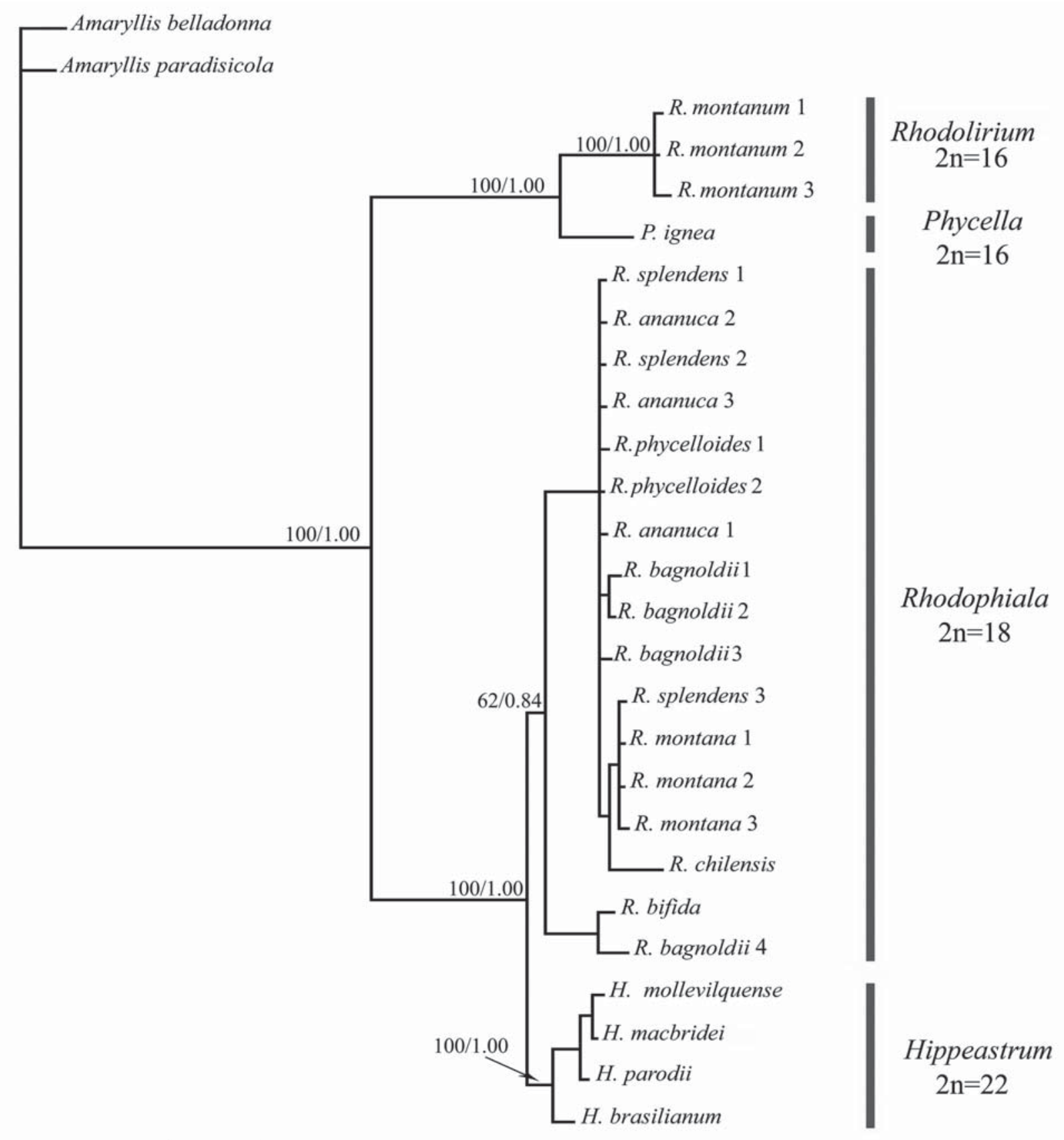

0,01

FIgURA 2. Árbol filogenético obtenido a partir de la región ITS de especies seleccionadas de Rhodolirium, Rhodophiala, Hippeastrum y Phycella. Cada taxa terminal corresponde a un individuo. Los valores sobre los nodos indican soporte de bootstrap (BS) y probabilidad $a$ posteriori (PP), respectivamente.

FIGURE 2. Phylogenetic tree of ITS region of selected species of Rhodolirium, Rhodophiala, Hippeastrum and Phycella. Each terminal taxa is an individual. Values above each node indicate bootstrap support (BS) and a posteriori probability (PP), respectively. 


\section{DISCUSIÓN}

Los resultados en cuanto a número y morfología cromosómica (Fig. 1) y topología del árbol filogenético (Fig. 2) son consistentes en las delimitaciones genéricas de Rhodolirium, Rhodophiala, Phycella e Hippeastrum.

Rhodophiala e Hippeastrum aparecen como grupos monofiléticos más emparentados entre sí que con respecto a los otros géneros analizados. Esto explicaría la inclusión de Rhodophiala en Hippeastrum en tratamientos taxonómicos clásicos (Baker 1878, Marticorena \& Quezada 1985). Meerow et al. (2000b) separaron estos géneros utilizando datos de la región ITS, lo cual es corroborado en el presente trabajo con las nuevas especies analizadas. Además esta separación está sustentada por diferencias en el número cromosómico, que en Hippeastrum es $2 \mathrm{n}=22$ y en Rhodophiala $2 \mathrm{n}=18$.

El otro grupo filogenéticamente emparentado lo constituyen Phycella y Rhodolirium, compartiendo igual número cromosómico $(2 \mathrm{n}=16)$, sin embargo los datos moleculares son robustos en separar ambas entidades (Fig 2). Además, las especies de Phycella se distinguen morfológicamente de Rhodolirium por su perigonio recto, tubular o estrechamente campanulado, caracteres diagnósticos del género (Ravenna 2003). Los análisis realizados en este trabajo confirman la separación del género Rhodolirium de Rhodophiala. Además de las diferencias moleculares y en el número cromosómico diploide, existen marcadas variaciones en la morfología cromosómica entre ambos géneros. La diferencia más notable entre Rhodophiala y Rhodolirium radica en que, en este último, los 2 pares de cromosomas de mayor tamaño corresponden a cromosomas metacéntricos (Fig. 1), característica observada en R. montanum (este trabajo), Rhodolirium speciosum (Herb.) Ravenna y Rhodolirium andicola (Poepp.) Ravenna (Baeza et al. 2009). Esta característica no se encuentra en los cariotipos de las especies de Rhodophiala descritos en este estudio ni en los descritos por Naranjo \& Poggio (2000) para R. bifida, $R$. elwesii y $R$. araucana.

Otra diferencia importante entre Rhodolirium y Rhodophiala se aprecia en el índice de asimetría cromosómica. Los índices de asimetría intracromosómica de Rhodophiala montana, $R$. splendens, $R$. bagnoldii y $R$. ananuca bordean los 0,6, diferenciándose de Rhodolirium montanum, cuyo índice de asimetría es de 0,46 que refleja una distinta morfología cromosómica. Romero Zarco (1986) indica que este índice es una buena forma de expresar la morfología cariotípica general en plantas. Naranjo \& Poggio (2000) determinaron índices de asimetría intracromosómica (A1) de 0,55 a 0,66 para Rhodophiala bifida, R. elwesii y R. araucana, diferenciándose claramente de $R$. montanum (= Rhodophiala rhodolirion) y Rhodolirium andicola (=Rhodophiala andicola) cuyos índices de asimetría fueron 0,36 y 0,23 respectivamente. Aunque el número cromosómico mayoritariamente reportado para Rhodophiala es de 2n = 18 (Palma-Rojas 2000, Baeza et al. 2006). Naranjo \& Poggio (2000) encontraron especies con dos citotipos, tal como ocurría para $R$. bifida $(2 \mathrm{n}=16 \mathrm{y}$ $2 \mathrm{n}=18$ ), y especies poliploides como sucede con $R$. elwesii $(\mathrm{n}=18,36,72)$. Estos citotipos no se encontraron en las especies estudiadas.

Con los datos obtenidos por este estudio se da soporte cromosómico y molecular para la separación de Rhodolirium de Rhodophiala, evidencia que apoya el tratamiento taxonómico basado principalmente en datos morfológicos propuesto por Ravenna (2003), para estos géneros y en donde la especie Rhodolirium montanum corresponde al tipo del género.

En especial la inferencia filogenética realizada en base a datos moleculares de la región ITS respalda a Rhodolirium como género válido. Por otra parte, la separación en el filograma de los distintos géneros analizados en grupos altamente soportados, confirma la utilidad de la información provista por la región ITS en las delimitaciones genéricas en Amaryllidaceae americanas, tal como anteriormente lo reportara Meerow et al. (2000b).

En síntesis, Rhodolirium es un género endémico de Chile y Argentina compuesto por la especie tipo del género $R$. montanum y las especies Rhodolirium andicola, $R$. chilense, $R$. fulgens, $R$. laetum y $R$. speciosum (Ravenna 2003). La principal diferencia morfológica entre los géneros Rhodolirium y Rhodophiala (género tratado como Myostemma Salisb. por Ravenna l.c.), es el grado de división del estigma, que en Rhodolirium es siempre capitado, en cambio en Rhodophiala es trífido (Baeza et al. 2009), el tubo del perigonio alargado (Philippi 1858) y un paraperigonio formado por escamas conspicuas, en muchas de sus especies. Cariológicamente las especies estudiadas del género Rhodolirium ( $R$. andicola, $R$. laetum, $R$. montanum y R. speciosum) (Naranjo \& Poggio 2000; Palma-Rojas 2000, Baeza et al. 2009) presentan un número cromosómico $2 \mathrm{n}=16$, número compartido por otros géneros endémicos de Chile de la familia Amaryllidaceae: Placea Miers, Phycella Lindl. y Traubia Moldenke (Flory 1968, Palma-Rojas 2000, Baeza \& Schrader 2004, Baeza et al. 2007a, 2009b), aunque la fórmula haploide es muy distinta (Baeza et al. 2009a), como también su morfología (Tabla II).

Sin duda, futuros estudios que combinen la morfología del cariotipo, los datos moleculares (ITS u otros) y la variación de los rasgos morfológicos de los géneros de Amaryllidaceae presentes en Chile dilucidarán la compleja taxonomía a nivel genérico de este grupo de plantas chilenas con potencial ornamental.

La correcta denominación de las entidades es necesaria para mejorar nuestro conocimiento de la biodiversidad (Valdecasas et al. 2008). El inadecuado tratamiento taxonómico de estos taxones refleja la falta de conocimiento de la biodiversidad de la familia Amarilidácea chilena, con el riesgo de que entidades diferentes y, probablemente, con potencialidades y atributos disímiles, permanezcan ocultas en taxones débilmente justificados. 


\section{AGRADECIMIENTOS}

Se agradece el financiamiento aportado por el proyecto FIA $\mathrm{N}^{\circ}$ BIOT-01-A-071 y al Dr. Marcelo Baeza (Universidad de Concepción) por el apoyo bibliográfico y comentarios.

\section{BIBLIOGRAFÍA}

Arroyo, S. C. \& D. F. Cutler. 1984. Evolutionary and taxonomic aspects of the internal morphology in Amaryllidaceae from South America and South Africa. Kew Bulletin 39: 467498.

Baeza, C.M. \& O. Schrader. 2004. Karyotype analysis of Placea amoena Phil. (Amaryllidaceae) by double fluorescence in situ hybridization. Caryologia 57: 200-205.

Baeza, C.M., O. Schrader \& I. Escobar. 2006. Estudio del cariotipo en Rhodophiala aff. advena (Ker-Gawl.) Traub de la VIII Región de Chile. Kurtziana 32(1-2): 45-51.

Baeza, C.M., E. Ruiz \& M. Negritto. 2007a. El número cromosómico de Phycella australis Ravenna (Amaryllidaceae). Gayana Botánica 64(1): 119-122.

Baeza, C.M., O. Schrader, A. Terrab, T. Stuessy, M. Rosas, E. Ruiz, M. Negritto \& E. Urtubey. 2007b. Recuentos cromosómicos en plantas que crecen en Chile. III. Gayana Botánica 64(2): 175-183.

Baeza, C.M., C. Mariangel, E. Ruiz \& M. Negritto. 2009. El cariotipo fundamental en Rhodolirium speciosum (Herb.) Ravenna y R. andicola (Poepp.) Ravenna (Amaryllidaceae). Gayana Botánica 66(1): 99-102.

BAKER, J.G. 1878. An enumeration and classification of the species of Hippeastrum. Journal of Botany 16: 79-85.

Baldwin, B., M. Sanderson, M. Porter, M. Wojciechowski, C. M. Campbell \& M. Donoghue. 1995. The ITS region of nuclear ribosomal DNA: a valuable source of evidence on angiosperm phylogeny. Annals of the Missouri Botanical Garden 82(2): 247-277.

DAYrat, B. 2005. Towards integrative taxonomy. Biological Journal of the Linnean Society 85: 407-415.

Felsenstein, J. 1985. Confidence limits on phylogenies: an approach using the bootstrap. Evolution 39: 783-791.

FLORY, W.S. 1968. Chromosome diversity in species, and in hybrids, of tribe Zephyrantheae. The Nucleus (Suppl.) 1868: 79-95.

Катон, K., K. Kuma, H. Тон \& T. Miyata. 2005. MAFFT version 5: improvement in accuracy of multiple sequence alignment. Nucleic Acids Research 33: 511-518.

Grant, J., A. Brown \& J. Grace. 1984. Cytological and isozyme diversity in Glycine tomentella Hayata (Leguminosae). Australian Journal of Botany 32: 665-667.

Levan, A., K. Fredga \& A. Sandberg. 1964. Nomenclature for centromeric position on chromosomes. Hereditas 52: 201220.

Marticorena, C. \& M. Quezada. 1985. Catálogo de la flora vascular de Chile. Gayana Botánica 42: 1-157.

Meerow, A.W. \& D.A. Snijman. 1998. Amaryllidaceae, In: K. Kubitzki (ed.). The families and genera of vascular plants III. Flowering plants. Monocotyledons. Lilianae (except Orchidaceae), pp. 83-110. Springer. Berlin.
Meerow, A., M. Fay, C. Guy, Q.-B. Li, F. Zaman \& M. Chase. 1999. Systematics of Amaryllidaceae based on cladistic analysis of plastid rbcL, and trmL-F sequence data. American Journal of Botany 86: 1325-1345.

Meerow, A., M. Fay, M. Chase, C. Guy, Q.- B. Li, D. Snijman \& S. L. YANG. 2000a. Phylogeny of Amaryllidaceae: molecules and morphology, en K.L. Wilson y D.A. Morrison (eds.). Monocots: Systematics and Evolution. pp. 372-386. CSIRO. Melbourne.

Meerow, A., C. Guy, Q.-B. Li \& S.L. Yang. 2000b. Phylogeny of the American Amaryllidaceae based on nrDNA ITS sequences. Systematic Botany 25: 708-726

Muñoz, M. \& A. Moreira. 2000. Géneros Endémicos Monocotiledóneas, Chile www.chlorischile.cl

Muñoz, M., R.Riegel \& P. SEEmann. 2006. Use of image citometry for the early screening of induced autopolyploids. Plant Breeding 125: 414-416.

NARANJo, C.A. \& B. AndradA. 1975. El cariotipo fundamental del género Hippeastrum Herb. (Amaryllidaceae) Darwiniana 19: 556-582.

Naranjo, C. \& L. Poggio. 2000. Karyotypes of five Rhodophiala species (Amaryllidaceae). Boletín de la Sociedad Argentina de Botánica 35(3-4): 335-343.

Olate, E. \& M. Bridgen. 2005. Techniques for the in vitro propagation of Rhodophiala and Leucocoryne spp. Acta Horticulturae 673: 335-339.

Palma-Rojas, C. 2000. Caracterización citogenética de los géneros Rhodophiala C.Presl y Phycella Lindl. (Amaryllidaceae). En: F. Schiappacasse \& P. Peñailillo (eds.), Los geófitos nativos y su importancia en la floricultura. Universidad de Talca. Chile. pp. 73-79.

Philippi, R.A. 1858. Plantarum novarum chilensium. Centuria quinta. Linnaea 29: 65-66.

Posada, D. 2008. jModelTest: Phylogenetic Model Averaging. Molecular Biology and Evolution 25: 1253-1256.

Posada, D. \& T.R. Buckley. 2004. Model selection and model averaging in phylogenetics: advantages of the AIC and Bayesian approaches over likelihood ratio tests. Systematic Biology 53: 793-808.

Ravenna, P. 2003. Elucidation and systematics of the Chilean genera of Amaryllidaceae. Botanica Australis 2: 1-20.

Romero Zarco, C. 1986. A new method for estimating karyotype asimmetry. Taxon 35: 526-530.

Ronquist, F. \& J.P. Huelsenbeck. 2003. MrBayes 3: Bayesian phylogenetic inference under mixed models. Bioinformatics 19: 1572-1574.

Schiappacasse, F., P. Peñailillo \& P. Yánez. 2002. Propagación de bulbosas chilenas ornamentales. Editorial Universidad de Talca. Talca. Chile. 65 pp.

Simpson, M.G. 2006. Plant Systematics. Amaryllidaceae. Elsevier Academic Press. Canada. pp. 169-171.

Strydom, A. 2005. Phylogenetic relationships in the family Amaryllidaceae. Thesis. Degree Ph.D., Faculty of Natural and Agricultural Sciences, Department of Plant Sciences, Genetics, University of the Free State. p. i-x +559 http:// etd.uovs.ac.za/ETD-db//theses/available/etd-09122006103922/unrestricted/StrydomA.pdf (accesada: 30 de noviembre de 2008).

Traub, H. 1956. The genera Rhodophiala C.Presl and Phycella Lindl.: key to the species and synonymy. Herbertia 12: 67-76. 
Traub, H. \& H. Moldenke. 1949. Amaryllidaceae: Tribe Amarylleae. The American Plant Life Society. Stanford. California. 194 pp.

Valdecasas, A., D. Williams \& Q. Wheller. 2008. 'Integrative taxonomy' then and now: a response to Dayrat (2005). Biological Journal of the Linnean Society, 93: 211-216.

Varghese, R., V. Chauhan \& A. Misra. 2003. Hypervariable spacer regions are good sites for developing specific PCRRFLP markers and PCR primers for screening actinorhizal symbionts. Journal of Bioscience 28: 437-442.

ZWICKL, D. J. 2006. Genetic algorithm approaches for the phylogenetic analysis of large biological sequence datasets under the maximum likelihood criterion. Ph.D. dissertation, The University of Texas at Austin. 115 pp.

Recibido: 20.05 .10

Aceptado: 18.10 .10 